Document downloaded from:

http://hdl.handle.net/10251/82092

This paper must be cited as:

Payri, R.; Salvador Rubio, FJ.; Gimeno, J.; Venegas Pereira, OH. (2016). A Technique to Match the Refractive Index of Different Diesel Fuels with the Refractive Index of Transparent Materials to Improve the Experimental Visualization. Experimental Techniques. 40(1):261269. doi:10.1007/s40799-016-0031-y.

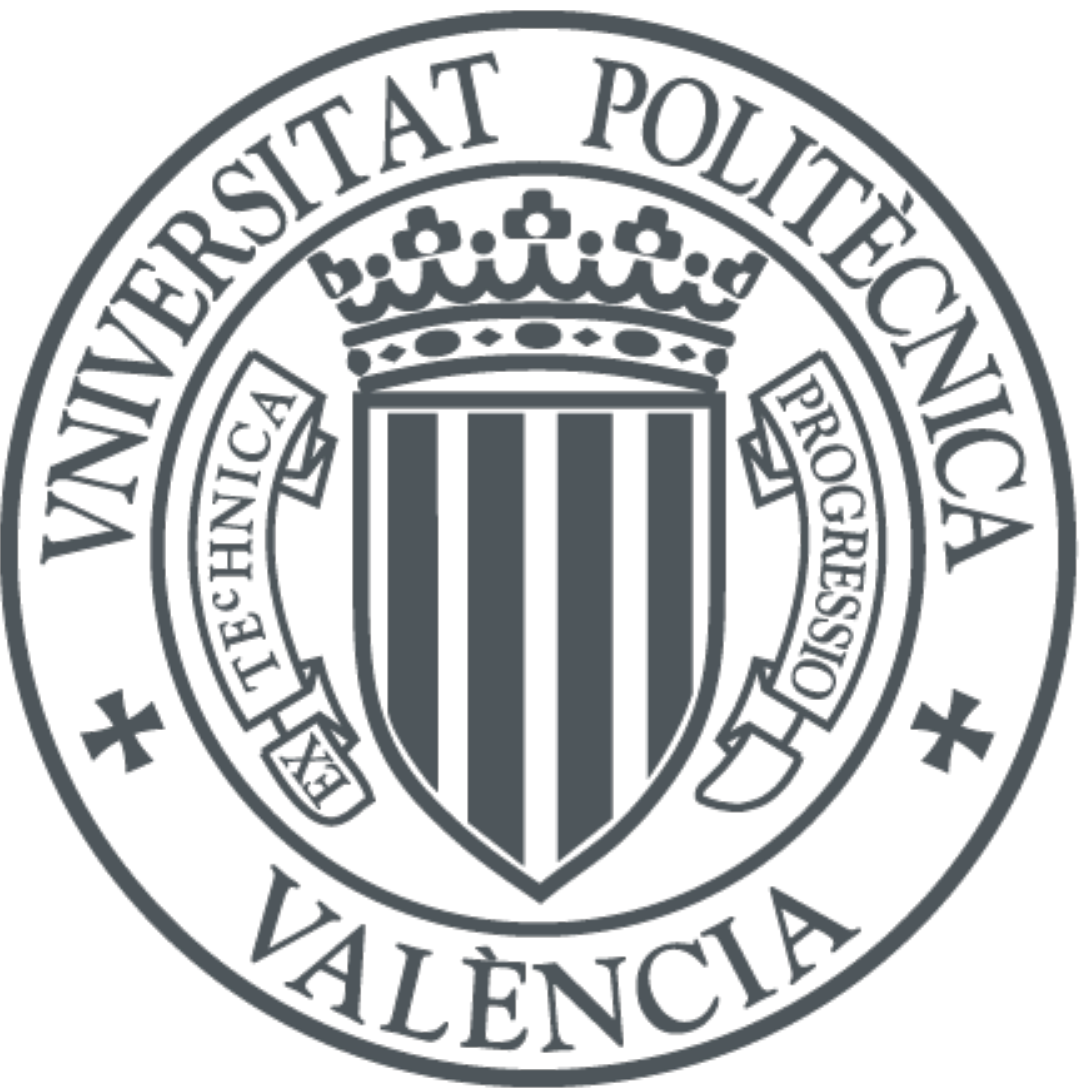

The final publication is available at

http://doi.org/10.1007/s40799-016-0031-y

Copyright Springer Verlag (Germany)

Additional Information 


\title{
A Technique to Match the Refractive Index of Different Diesel Fuels with the Refractive Index of Transparent Materials to Improve the Experimental Visualization
}

\author{
R. Payri, F.J. Salvador, J. Gimeno, O. Venegas \\ CMT-Motores Térmicos. Universitat Politècnica de València. Spain.
}

\begin{abstract}
In the present paper a technique to match the refractive index $(n)$ of different diesel fuels with the refractive index of transparent materials is presented with the aim to improve the flow visualization inside transparent nozzles. For this purpose, a technique based on the variation of the angle of a laser beam passing through the tested fluid and the transparent material was used. The validation of this technique was performed by measuring the refractive index of known fluids ( $\mathrm{n}$-decane and n-hexadecane) and comparing them with literature ones. After this, the refractive index was determined for three different diesel fuels: commercial diesel, a rapeseed methyl ester biodiesel (RME) and cold start diesel. The uncertainty of the measurements represents a relative error respect to the mean of only $0.1 \%$. Next, to equal the refractive index of each fuel with the refractive index of the transparent material (fused silica nozzle), a doping process was performed with 1-methylnaphthalene and $\mathrm{n}$ hexadecane at different concentrations. The main advantage of applying this technique in visualization measurements is to obtain additionally the fuel refractive index and match this with the transparent nozzle under real conditions without requiring additional equipment such as refractometers.
\end{abstract}

KEYWORDS: refractive index, refraction angle, transparent nozzle, diesel, cavitation, RME, fused silica.

\section{INTRODUCTION}

The reduction of emissions and fuel consumption is currently one of the most important subjects in the automotive sector and society in general. The number of research activities focused on the fundamental understanding of the internal combustion engine processes has increased rapidly.

Cite as:

Payri, R.; Salvador, F.; Gimeno, J. \& Venegas, O., "A Technique to Match the Refractive Index of Different Diesel Fuels with the Refractive Index of Transparent Materials to Improve the Experimental Visualization", Experimental Techniques, (2016) Vol. 40(1), pp. 261-269, doi: 10.1007/s40799-016-0031-y 
One of the areas of interest in diesel engines is the study of nozzle flow behavior in fuel injectors. As it is well known through previous studies from other authors [1-4], the flow behavior inside the hole is strongly related to the behavior of the spray at the exit of the hole and its interaction with the air, affecting mixing and combustion process.

Many researchers have worked with diverse visualization techniques of the spray at the exit of the nozzle to obtain information about the characteristic parameters of the spray, such as the angle and penetration. However, other researches [5-11] point to the visualization of the flow inside the nozzle with the goal to observe the cavitation phenomenon and its influence in the spray development. In these researches, different nozzle models have been developed with optical materials that allow the visualization inside the hole such as quartz (fused silica, $\mathrm{SiO}_{2}$ ) and methacrylate.

One of the big problems to achieve an optimal visualization of the flow inside the hole in transparent nozzles is the image distortion as a result of the refraction of the light passing through different media. The goal of this paper is getting to know the refractive index of the fluid passing inside the hole and then be able to equal its refractive index with the refractive index of the nozzle in order to prevent the refraction of light between the fluid and the solid material and thus ensure a correct visualization of the inner flow.

To do so, an assembly has been made composed by a laser and a CCD camera with which the refractive index of the fluid can be measured and matched with the refractive index of the transparent material. Although the refractive index could be measured using any device designed for this purpose, the advantage that the proposed assembly presents is that it allows matching the refractive index of the fluid with the transparent material using the same elements that will be employed in the experimental visualization (laser, CCD camera and transparent nozzle) and the same conditions at which tests are performed. Thus, the presented technique can be tested and applied easily when a laser and a CCD camera are available.

The present study is divided into three stages: In the first stage, the calibration of the assembly is performed using test fluids. In the second stage, the refractive index for three diesel fuels is found. Finally, the refractive index of the tested fluids will be equaled by doping them until reaching the value of the refractive index of the transparent material.

This paper is structured in four sections. First, a review of the concepts used in the calculation and definition of the refractive index is carried out. Second, the experimental facilities and methodology are described; in this 
section the processing of each captured image is also explained. In the third section, the results of test fluids for assembly calibration are presented, then the results for the fuels in pure state and finally for the doped fuels to reach the value of the refraction index of the transparent material. Finally, some general conclusions and possible future works will be established.

\section{REFRACTIVE INDEX THEORY}

The ratio between the speed of light in vacuum $(c)$ and the speed of light in a transparent medium $(v)$ is named refractive index. Since the light frequency does not change as passing from one medium to another, the wavelength changes and becomes smaller when the medium has a higher refractive index as shown Eq.(1).

$$
\text { (1) } \quad n=\frac{c}{v}=\frac{\lambda_{0} \cdot f}{\lambda \cdot f}=\frac{\lambda_{0}}{\lambda}
$$

Where $\lambda_{0}$ is the wavelength of the light at a certain frequency $(f)$ that propagates in the vacuum and $\lambda$ the wavelength that propagates in the medium at the same frequency ( $₫$ Error! No se encuentra el origen de la referencia.).

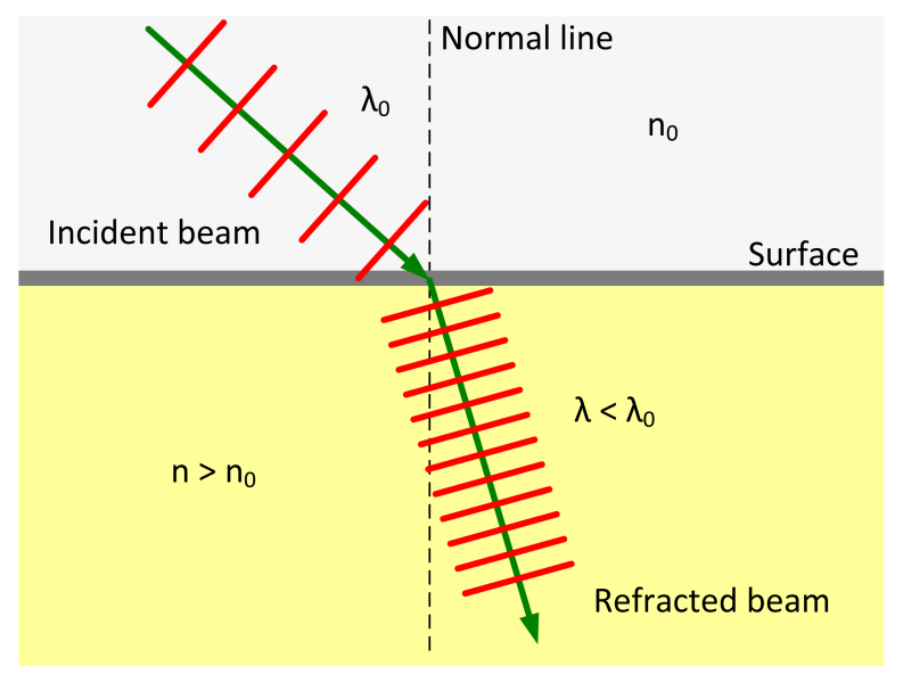

Figure 1. Wavelength in the vacuum and another medium at same frequency

To calculate the refraction angle of the light going through the separation surface between two media, Snell's law, defined by Eq.(2), is usually used.

$$
\text { (2) } \quad n_{1} \cdot \sin \theta_{1}=n_{2} \cdot \sin \theta_{2}
$$

Where $n_{1}$ and $n_{2}$ are the refractive indexes of the media and $\theta_{1}$ and $\theta_{2}$ are the incidence and refraction angles formed with a normal line to the surface.

The refractive index of a medium depends upon the wavelength of the light being transmitted. Each wavelength of light will be subject to a different refractive index [12]. Table 1 gives values for the refractive index of materials at a temperature of $20^{\circ} \mathrm{C}$ and standard pressure of $0.1 \mathrm{MPa}$. These values are presented for different wavelengths. 


\begin{tabular}{cccc}
\hline \hline Materials & $\boldsymbol{n}_{\boldsymbol{F}}(\mathbf{4 8 6 . 1 3 n m})$ & $\boldsymbol{n}_{\boldsymbol{D}}(\mathbf{5 8 9 . 2 6 n m})$ & $\boldsymbol{n}_{\boldsymbol{C}}(\mathbf{6 5 6 . 2 8 n m})$ \\
\hline Air [14] & 1,00027936 & 1,00027715 & 1,00027625 \\
Fuse silica (SiO2) [13, 15] & 1,46313 & 1,4584 & 1,45637 \\
n-decane [16] & 1,41660 & 1,41160 & 1,40940 \\
n-hexadecane [17] & 1,43985 & 1,43449 & 1,43225 \\
\hline \hline
\end{tabular}

Table 1. Refractive index at different wavelengths

In normal dispersion, the refractive index increases for shorter wavelength of light [12]. Figure 2 illustrates the refractive index as a function of the wavelength for the quartz (fused silica). It is often desired to have a mathematical representation of the index as a function of wavelength. A considerable number of models exist just for this purpose [12-19]. Perhaps the best known, and most widely used, is the Sellmeier form as shown in Eq.(3).

(3) $\quad n^{2}(\lambda)=1+\frac{B_{1} \cdot \lambda^{2}}{\lambda^{2}-C_{1}}+\frac{B_{2} \cdot \lambda^{2}}{\lambda^{2}-C_{2}}+\frac{B_{3} \cdot \lambda^{2}}{\lambda^{2}-C_{3}}$

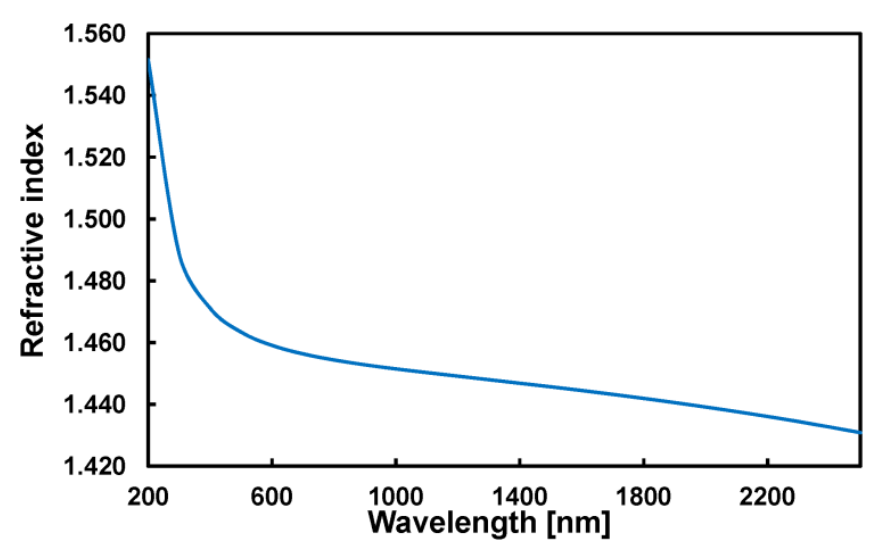

Figure 2. Fused silica chromatic dispersion curve

Where $\lambda$ is the wavelength in micrometers and the constants $B_{1}, B_{2}, B_{3}, C_{1}, C_{2}$ and $C_{3}$ are called Sellmeier coefficients of each optical material. Table 2 shows Sellmeier coefficients for the fused silica [13].

\begin{tabular}{l|l}
\hline \hline $\boldsymbol{B}_{1}$ & $6.96166300 \mathrm{E}-01$ \\
$\boldsymbol{B}_{2}$ & $4.07942600 \mathrm{E}-01$ \\
$\boldsymbol{B}_{3}$ & $8.97479400 \mathrm{E}-01$ \\
$\boldsymbol{C}_{\boldsymbol{1}}$ & $4.67914826 \mathrm{E}-03$ \\
$\boldsymbol{C}_{2}$ & $1.35120631 \mathrm{E}-02$ \\
$\boldsymbol{C}_{3}$ & $9.79340025 \mathrm{E}+01$ \\
\hline
\end{tabular}

Table 2. Sellmeier coefficients for fused silica [13]

Other numerous dispersion models have been developed and are presented elsewhere [12-19]. For organic compounds Forziati [20] has shown that the optical dispersion can be represented accurately by the Hartmann dispersion formula [19, 21] shown in Eq.(4).

$$
\text { (4) } \quad n(\lambda)=A+\frac{B}{\lambda-C}
$$

Where $\lambda$ is the wavelength and $A, B$ and $C$ are the Hartmann constants and are defined as: 


$$
\begin{gathered}
B \equiv \frac{n_{1}-n_{2}}{\frac{1}{\lambda_{1}-C}-\frac{1}{\lambda_{2}-C}} \\
A \equiv n_{1}-\frac{B}{\lambda_{1}-C}
\end{gathered}
$$

where $n_{1}, n_{2}$ and $n_{3}$ are the refractive indices of the organic compound at different wavelengths $\lambda_{1}, \lambda_{2}$ and $\lambda_{3}$ respectively.

\section{EXPERIMENTAL SET UP}

To carry out this study, an assembly as shown in Figure 3., has been developed. The aim of this assembly is to measure the angles of incidence and refraction of the laser beam passing through the fluid and the transparent nozzle and so find the refractive index of the fluid. Below, each one of its elements is described.

- Fused silica piece with a refractive index known. This material is used as reference to know the refractive index of the fluids to be studied. Actually the piece is a transparent nozzle used in the visualization tests.

- $100 \mathrm{ml}$ cuvette which contains the fluids and the fused silica nozzle.

- Continuous Argon Laser with a wavelength of $514.5 \mathrm{~nm}$. Its function is to pass a beam of light through the fluid and the transparent nozzle.

- Goniometer, used to change the inclination angle of the beam.

- A CCD Pixel-fly camera accompanied by an acquisition system to save the captured images of the beam going through the fluid and the nozzle.

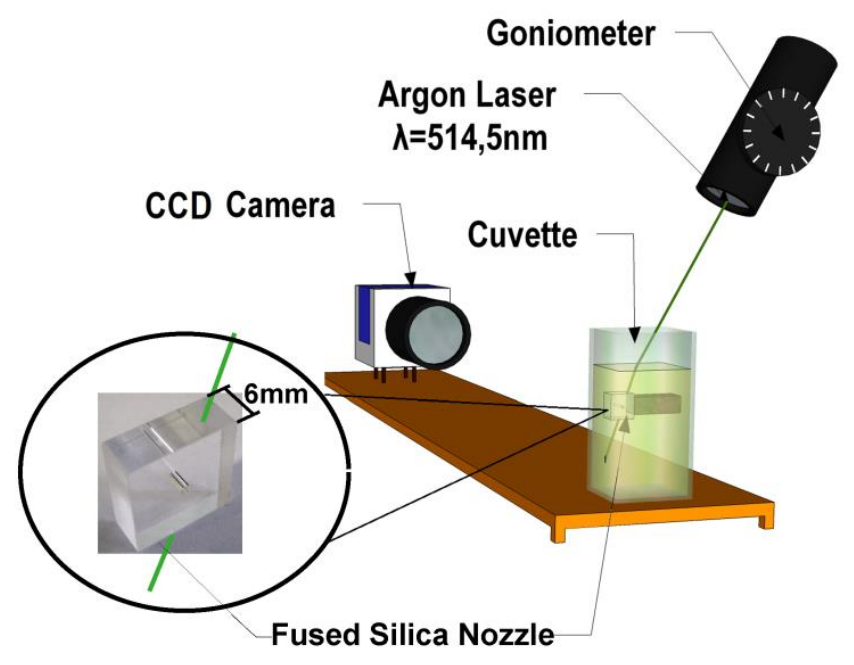

Figure 3. Assembly scheme to measure the refractive index

On the other hand, different fluids were used to carry out this experimental technique:

- Test fluids to validate the technique: $\mathrm{n}$-decane and $\mathrm{n}$-hexadecane.

- Fluids to be measured and for which the refractive index will be found: commercial diesel fuel, a biodiesel RME 100\% (Rapeseed Methyl Ester) and Artic fuel (diesel for cold start). 
- Doping fluids to equal the refractive index of the measured fuels with the fused silica nozzle: $n$-hexadecane and 1-methylnaphthalene.

To take the measurements, the following methodology is used: as prior steps to images capturing, the laser angle inclination is regulated with the goniometer and the fluid temperature is maintained between $18{ }^{\circ} \mathrm{C}$ and $22^{\circ} \mathrm{C}$. The beam passes through the transparent nozzle without touching the injection hole in the middle of this. Then, for each component or mixture 100 images are taken using an exposure time of $240 \mathrm{~ms}$. For all tests the power of the laser was kept stable at $26 \mathrm{~mW}$.

\section{Image processing}

Once images are taken, they are processed using a purpose-made algorithm. With this processing the angles of incidence and refraction between the fluid and the fused silica nozzle are obtained.

The processing consists in finding two contours of the beam above and below the transparent nozzle and then midlines to these contours are drawn as shown in Figure 4; after getting the midlines, the function looks for an intersection point with the transparent nozzle surface. By connecting these intersection points, the refraction angle of the nozzle $\left(\theta_{2}\right)$ is finally obtained. The same process is applied for each image, getting processed images as shown in Figure 5.
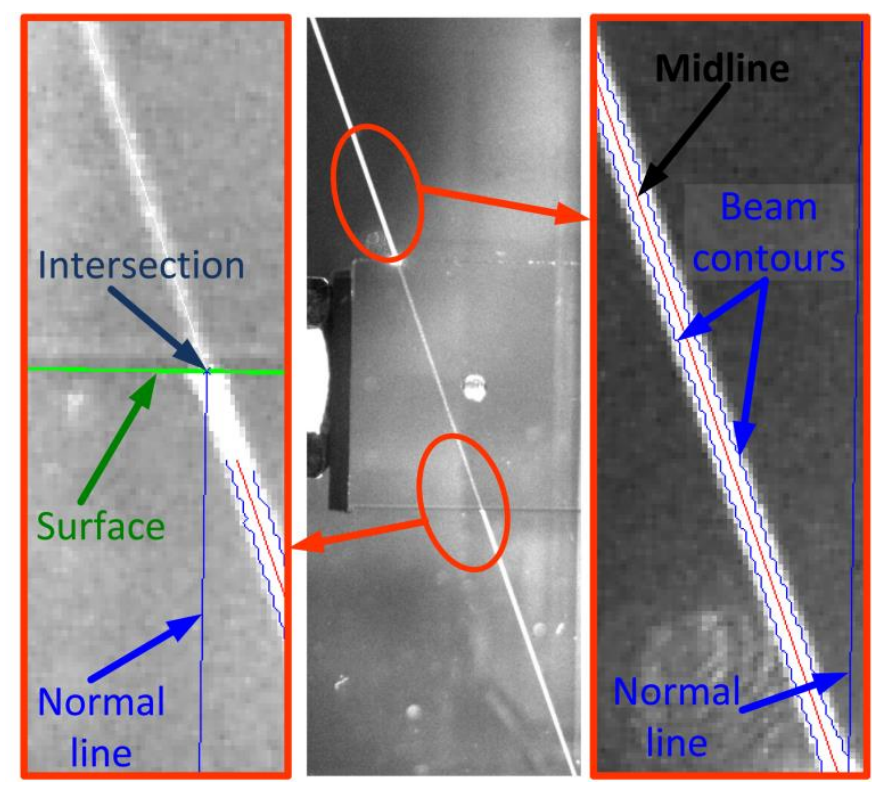

Figure 4. Original image (middle) and processed image (left and right) 


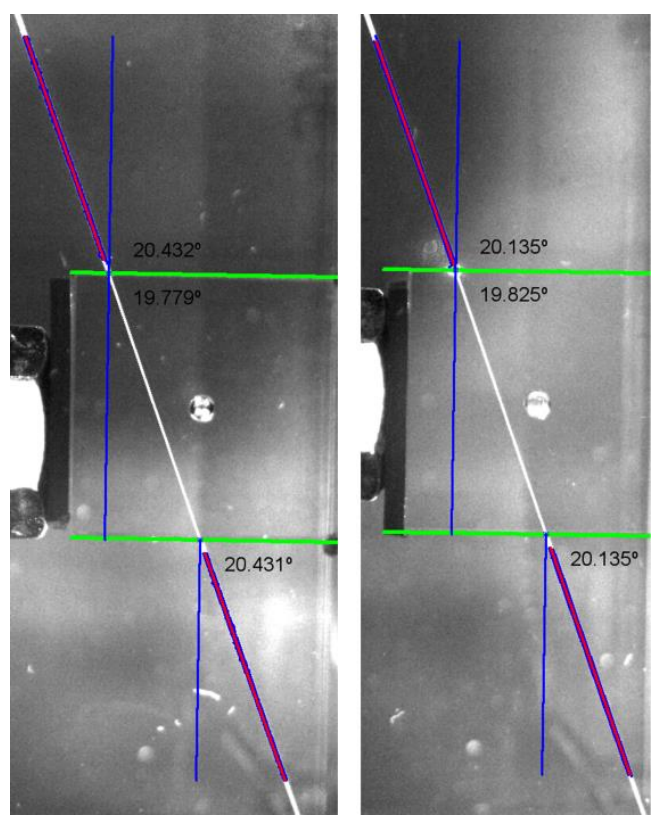

Figure 5. Processed images for n-decane (left) and n-hexadecane (right)

Once the angles of incidence $\theta_{1}$ and refraction $\theta_{2}$ in the nozzle are known, it is possible to find the refractive index of the fluid. First, since the wavelength of the beam through the air is known $(514.5 \mathrm{~nm})$, Sellmeier's dispersion model, shown in Eq.(3)., can be applied to obtain the refractive index of the nozzle.

With the refractive index of the fused silica at a given wavelength (wavelength of the laser beam), and with the angles obtained from the processing, it is possible to determine the refractive index of test fuels and all other measured fluids.

\section{RESULTS}

With the aim to choose an angle of inclination where the dispersion in the measurements is as smaller as possible, several tests are conducted varying the angle of the goniometer from 15 to 30 degrees. The fluid used for these measurements was commercial diesel. After processing the images it is observed that as the inclination angle of the laser increases the measurements dispersion decreases, as shown in Figure 6. This figure shows different boxplots for each angle tested. This way, it is decided to work from now on with an inclination angle of 30 degrees, since the dispersion is lower. No tests have been conducted with angles higher than 30 degrees due to the restrictions of the assembly. 


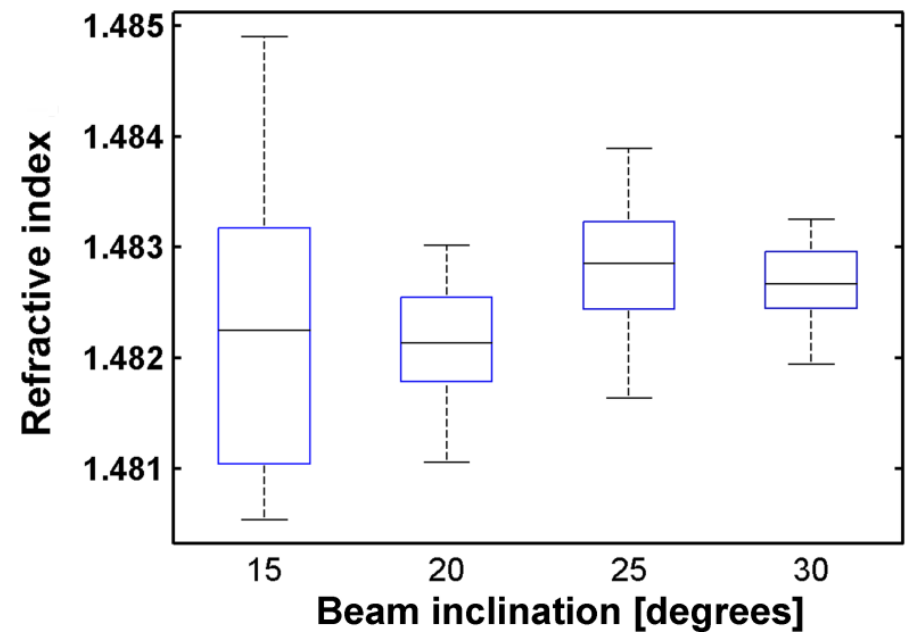

Figure 6. Refractive index dispersion of commercial gasoil at different beam inclination angles

\section{Calibration}

Once the beam angle has been fixed, the measurements with test fluids ( $\mathrm{n}$-decane and $\mathrm{n}$-hexadecane) are carried out to calibrate the assembly and validate the technique. In the boxplots in Figure 7 the dispersion for each test fluid is shown. In order to compare the median of the resulting values to those found in the literature, values from Table 1 are used and Equations (5), (6) and (7) are applied to obtain the Hartmann constants for each test fluid. The value of each constant is tabulated in Table 3.

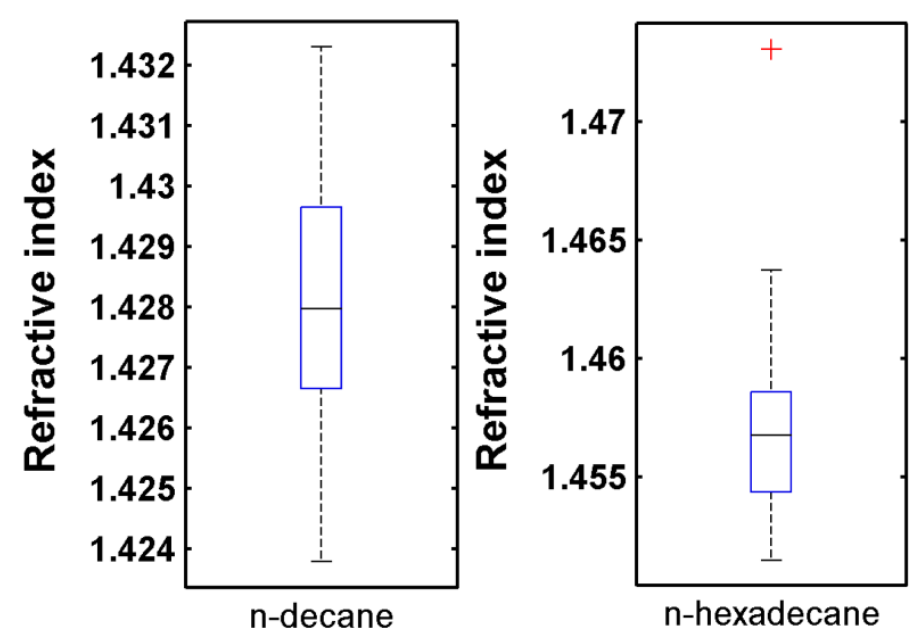

Figure 7. Experimental measurements dispersion for test fluids

\begin{tabular}{ccc}
\hline \hline Test fluid & Hartmann constant & Value \\
\hline \multirow{2}{*}{ n-decane } & C & 0,12939 \\
& B & 0,00795 \\
A & 1,39430 \\
\hline \multirow{2}{*}{-hexadecane } & C & 0,17956 \\
& B & 0,00653 \\
\hline \hline & A & 1,41856 \\
\hline
\end{tabular}

Once Hartmann constants are known, using refractive indices at specific wavelengths obtained from the literature (Table 1), the theoretical refractive index of the test fluids al $514.5 \mathrm{~nm}$ can be found using the Eq.(4). In 
this way, the experimental refractive index can be compared to the theoretical refractive index for the same wavelength.

In Table 4 the values obtained experimentally are compared to the found in literature. It can be seen that the real and experimental values show a difference less than 0.001 in the refractive index value. This difference is called uncertainty due to calibration of the equipment $\left(U_{\text {calibration }}\right)$ and will be taken into account later in the calculation of uncertainty for measured fuels.

\begin{tabular}{ccc}
\hline \hline Test fluid & Theoretical & $\begin{array}{c}\text { Experimental } \\
\text { median }\end{array}$ \\
\hline n-decane & 1,415 & 1,414 \\
n-hexadecane & 1,438 & 1,439 \\
\hline
\end{tabular}

Table 4. Comparison between experimental and theoretical $n$ for test fluids at $514,5 \mathrm{~nm}$

Since the uncertainty due to calibration for both test fluids is almost the same, any of them can be taken to find the uncertainty of the measured fuels; in this work the $n$-hexadecane is chosen as pattern fluid because its properties are more similar to the measured fuels.

\section{Refractive index of target fuels}

Following a similar procedure for the target fluids (commercial fuel, RME and Artic), the refractive index was obtained. To find the standard combined uncertainty, the affecting factors are considered as shown in Eq.(8).

$$
\text { (8) } U=\sqrt{{U_{m}}^{2}+U_{c}^{2}+U_{T}^{2}}
$$

Where $U_{m}$ is the uncertainty associated to the measure itself, $U_{c}$ the calibration uncertainty and $U_{T}$ the uncertainty associated to temperature.

The uncertainty associated to the measurement shown in Eq.(9) takes into account the uncertainty due to repeatability of the measures and the resolution of the elements used in the assembly. This is related to the measurements repeatability which is the standard deviation of the results. Thus, the uncertainty due to the measurements is the dispersion of the mean.

$$
\text { (9) } U_{m}=\sqrt{U_{\text {repeatability }}^{2}}
$$

The uncertainty due to calibration shown in Eq.(10) considers the uncertainty in the experimental measurement of the pattern (n-hexadecane), the uncertainty of the pattern itself $(0.00006$ [17]) and the uncertainty due to calibration of the equipment, which is the difference between theoretical and experimental data (close to 0.001 according to Table 4).

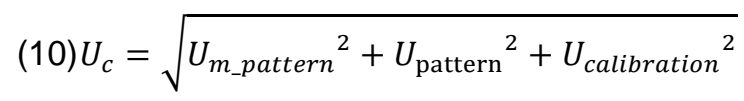


Finally, assuming a rectangular distribution, the uncertainty due to temperature is calculated according to Eq.(11).

$$
\text { (11) } U_{T}=\frac{S \cdot \Delta T}{\sqrt{3}}
$$

Where $S$ is the sensibility to temperature change of the n-hexadecane $(0.00040$ [17]) and $\Delta T$ the maximum difference that can exist between the experimental temperature and the temperature at which the pattern was measured $\left(\Delta T=(22-20)^{\circ} \mathrm{C}=2^{\circ} \mathrm{C}\right)$.

The last step is to calculate the total expanded uncertainty $\left(U_{e}\right)$. To do so, the standard uncertainty must be multiplied by a coverage factor $K$ as shown in Eq.(12).

$$
\text { (12) } U_{e}=K \cdot U
$$

Usually a $\mathrm{K}=2$ is considered, so there is a $95 \%$ probability that the real refractive index is contained in the interval $R I \pm U_{e}$. Table 5 summarizes the values obtained for the measured fuels with the respective uncertainty and wavelength at which the beam passes through the fluid.

\begin{tabular}{ccc}
\hline \hline Fuel & $\begin{array}{c}\text { Refractive } \\
\text { index }\end{array}$ & $\begin{array}{c}\text { Expanded } \\
\text { uncertainty }\end{array}$ \\
\hline $\begin{array}{c}\text { Commercial } \\
\text { diesel }\end{array}$ & 1,468 & $\pm 0,002$ \\
RME & 1,457 & $\pm 0,002$ \\
Artic & 1,462 & $\pm 0,002$
\end{tabular}

Table $\overline{\overline{5 . \text { Refractive index obtained for target fuels at } 51}} 4,5 \mathrm{~nm}$

\section{Equaling $\boldsymbol{n}$ of the measured fuels with the $n$ of fused silica}

To equal the refractive index of these fuels with the fused silica refractive index, 1-methylnaphthalene or $\mathrm{n}$ hexadecane is added depending on whether the measured fuel has a refractive index greater or lower than the fused silica. According to the dispersion of Sellmeier, shown in Eq.(3), the fused silica has a refractive index of 1.46158 at wavelength of $514.5 \mathrm{~nm}$. Figure 8 shows the change in the refractive index as the concentration of the doping fluid changes. 


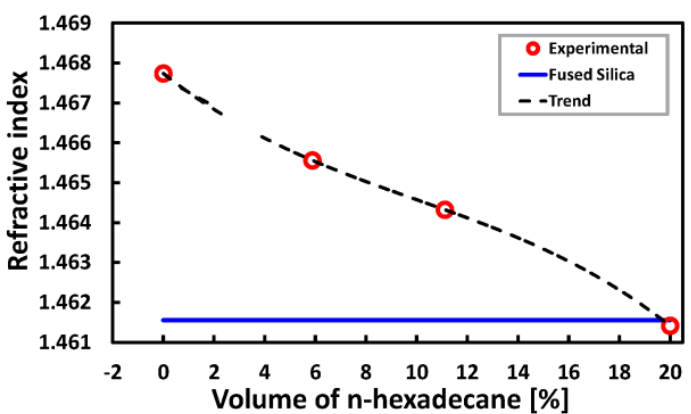

(a)

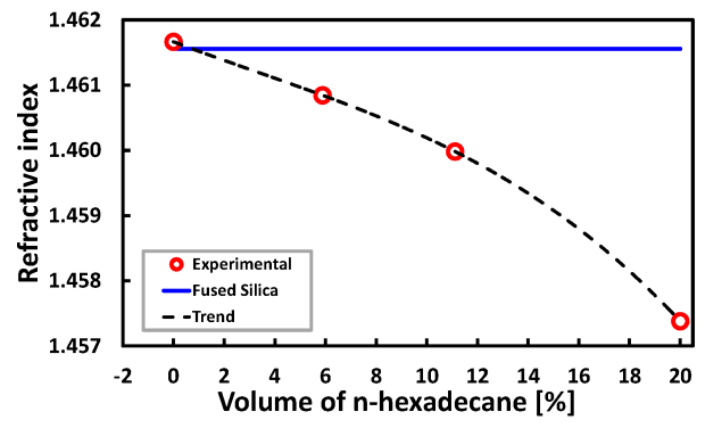

(c)

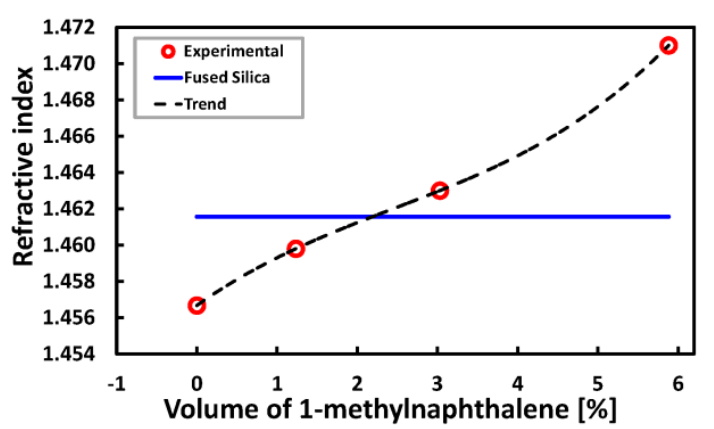

(b)

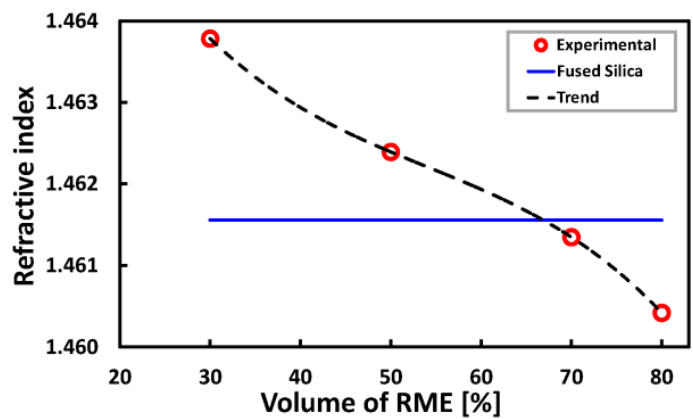

(d)

Figure 8. Variation of $\mathbf{n}$ at different concentrations of doping fluid for target fuels: (a) and (d) Commercial gasoil, (b) RME and (c) Artic

As the refractive index of commercial diesel is above the refractive index of the fused silica and the RME refractive index is lower, a last test is performed mixing the commercial diesel with RME. Figure 8(d) shows the trend of the refractive index as the concentration varies.

Figure 9 shows an example of the variation of the angles of incidence and refraction when the RME concentration changes (similarly occurs for other fuels). It can be seen that as the concentration of RME rises, the difference between the angles becomes smaller (for 30\% and 50\%). From 70\% this difference increases again because the refractive index of the fluid is lower than the fused silica.
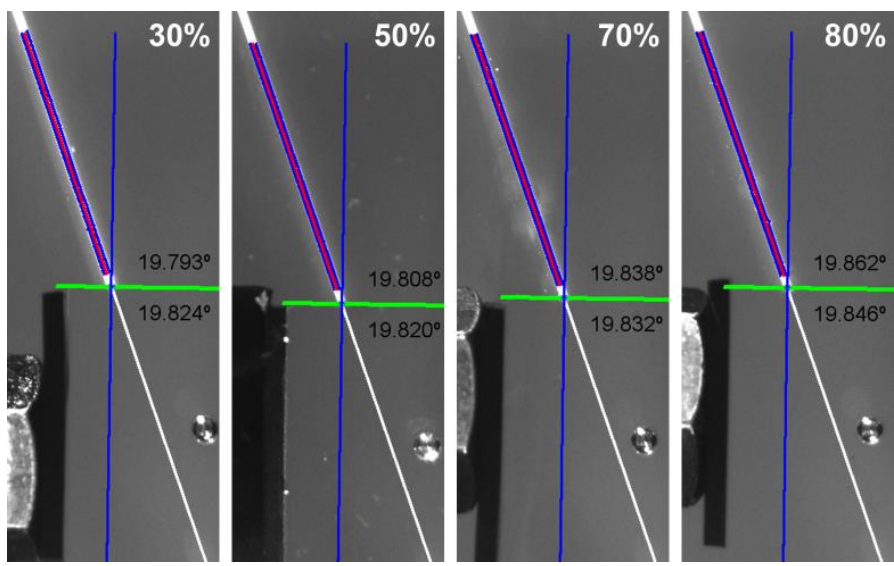

Figure 9. Incidence and refractive angles for commercial gasoil at different concentrations of RME 
The trend of the curves from the Figure 8 fits a third order polynomial (13). ¡Error! No se encuentra el origen de la referencia. shows the coefficients of the polynomial for each case.

$$
\text { (13) } R I=a_{3} \cdot V^{3}+a_{2} \cdot V^{2}+a_{1} \cdot V+a_{0}
$$

\begin{tabular}{cccccc}
\hline \hline (14) Fuel & $\boldsymbol{a}_{\mathbf{3}}$ & $\boldsymbol{a}_{\mathbf{2}}$ & $\boldsymbol{a}_{\mathbf{1}}$ & $\boldsymbol{a}_{\mathbf{0}}$ & $\mathbf{R}^{\mathbf{2}}$ \\
\hline Commercial diesel & $-9 \mathrm{E}-07$ & $3 \mathrm{E}-05$ & $-0,0005$ & 1,4677 & 0,99 \\
RME & $8 \mathrm{E}-05$ & $-6 \mathrm{E}-04$ & 0,0031 & 1,4567 & 0,99 \\
Artic & $-3 \mathrm{E}-07$ & $3 \mathrm{E}-06$ & $-0,0001$ & 1,4616 & 0,99 \\
Commercial diesel & $-3 \mathrm{E}-08$ & $5 \mathrm{E}-06$ & $-0,0004$ & 1,4703 & 0,99 \\
\hline \hline \multicolumn{5}{c}{ Table 6. Trend line coefficients }
\end{tabular}

From the results obtained, it can be observed that the commercial diesel has a greater refractive index than the fused silica; the Artic has a similar value and the RME is below it. Table 7 summarizes the necessary concentration in doping or mixing fluid volume to equal the refractive index of the test fluids with the fused silica refractive index.

\begin{tabular}{ccc}
\hline \hline Fuel & Doping or mixing fluid & Volume [\%] \\
\hline Commercial diesel & n-hexadecane & 19,72 \\
RME & 1-methylnaphthalene & 2,19 \\
Artic & n-hexadecane & 0,75 \\
Commercial diesel & RME & 66,03
\end{tabular}

Table 7. Necessary amount of doping or mixing fluid to equal the $n$ of fuel with the $n$ of fused silica

Finally from Figure 8 and Table 7 it can be seen that the volume of $n$-hexadecane necessary to match the refractive index of the commercial gasoil with the fused silica is very high and can affect considerably its properties. Instead, when we mix RME with commercial gasoil, although the mix has a high content of RME it still has the typical properties of a diesel fuel (like density, vapor pressure and superficial tension) since both RME and commercial gasoil are diesel fuels used for the same purpose and with very similar properties. On the other hand, to match the refractive index of the Artic and the RME with the fused silica index, the required amount of dopant fluid is very low and therefore acceptable. In conclusion, from the four tested mixtures, three are acceptable.

\section{CONCLUSIONS}

The aim of this paper was to present a technique to match the refractive index of different diesel fuels with the refractive index of transparent materials. In this document a transparent nozzle of fused silica was used as transparent material. This study was made with the goal to contribute to improving the flow visualization into transparent nozzles, whereas an alternative technique to measure the refractive index is presented. 
The refractive index of the fuels previously tested was matched to the refractive index of the solid material (fused silica nozzle). To do this, n-hexadecane and 1-methylnaphthalene were used to decrease or increase the refractive index. This way, it was possible to observe the variation referred to the refractive index for each fuel and, according to the trend of the tested points, to obtain the required concentration to equal the refractive index with the refractive index of the fused silica. In figure 9 the difference between incidence and refraction angles to varying the mixture could be appreciated, showing that when this difference is smaller, the refractive index of the fluid is more similar to the refractive index of the transparent nozzle.

To perform all measurements, an assembly including a continuous laser of $514.5 \mathrm{~nm}$ and a CCD camera was made. This assembly allows taking images of the beam passing through the fluid and the solid material. With these images and posterior processing, it was possible to determine the angles of incidence and refraction between the fuel and the solid material. Besides, it was also possible to know the change in the beam wavelength as it goes through the air $(514.5 \mathrm{~nm})$, the fluid (different for each fuel) and the solid (352.1nm).

Knowing the angles of incidence and refraction and also the refractive index of the solid material it was possible to determine the refractive index of the different tested fluids. The uncertainty of the measurements was calculated taking into account the factors that affect the measure such as temperature, calibration and the deviation of the measures. It can be seen that this values of uncertainty are the same for all tested fluids and they represent a relative error respect to the mean of only $0.1 \%$.

The technique shown in this document is useful to obtain images of the internal flow in transparent nozzles with good quality by adjusting the refractive index on-site without resorting to additional devices. In the current document, the fluid temperature was kept constant and atmospheric pressure. However, a major advantage of this technique is its use to actual operating conditions, where varies the pressure (to $5 \mathrm{MPa}$ ) and the temperature (to $40^{\circ} \mathrm{C}$ ), which is very difficult to achieve with apparatus for the measurement of refractive index.

\section{ACKNOWLEDGMENTS}

The authors would like to José Enrique del Rey* for his collaboration in the experimental measurements. This work was carried in the frame of OPTICOMB-ENGINE Project; reference TRA2007-67961-C03-01/AUT with the support of the National R\&D of the Government of Spain. $\left(^{*}\right)$ From CMT-Motores Térmicos. Universitat Politècnica de València. 


\section{CONTACT}

Dr. Jaime Gimeno, jaigigar@mot.upv.es

CMT-Motores Térmicos, Universitat Politècnica de València.

\section{NOMENCLATURE}

$$
a_{0}, a_{1}, a_{2}, a_{3} \quad \text { Coefficients of the polynomial trend }
$$

$\boldsymbol{A}, \boldsymbol{B}, \boldsymbol{C} \quad$ Hartmann constants

$B_{1}, B_{2}, B_{3}, C_{1}, C_{2}, C_{3} \quad$ Sellmeier coefficients

$c$

$f$

$\boldsymbol{K}$

$n$

$n_{1}$

$n_{2}$

$S$

$\boldsymbol{U}$

$U_{c}$

$U_{\text {calibration }}$

$U_{e}$

$\boldsymbol{U}_{m}$

$U_{m \_p a t t e r n}$

$U_{\text {pattern }}$

$U_{\text {repeatability }}$

$\boldsymbol{U}_{T}$

$\boldsymbol{v}$

V

Greek Symbols

$\Delta T$

$\lambda_{0}$

$\lambda$

$\boldsymbol{\theta}_{0}$ $\lambda$
Speed of light in vacuum

Light frequency

Coverage factor

Refractive index

Refractive index in medium 1

Refractive index in medium 2

Sensibility to temperature change of pattern

Standard combined uncertainty

Calibration uncertainty

Correction due to calibration of the equipment

Total expanded uncertainty

Uncertainty associated to the measure

Uncertainty associated to the measure of pattern

Uncertainty of the pattern

Uncertainty due to repeatability of the measures

Uncertainty due to temperature

Speed of light in a transparent medium

Percentage concentration by volume of dopant fluid
Maximum difference between the experimental temperature and the temperature at which the pattern was measured

Wavelength of the light in the vacuum

Wavelength of the light

Incident angle in the fuel 


$\begin{array}{ll}\boldsymbol{\theta}_{1} & \text { Incident angle in the nozzle } \\ \boldsymbol{\theta}_{2} & \text { Refraction angle in the nozzle } \\ \boldsymbol{\theta}_{2 \_ \text {crit }} & \text { Critical angle }\end{array}$

\section{REFERENCES}

1. Payri, R., Bermúdez, V., Salvador, F.J., and Plazas, A.H. Study of the influence of nozzle seat type on injection rate and spray behavior. Proc. ImechE, vol. 219 Part D: J. Automobile Engineering, pp. 677-689, 2005.

2. Afzal, H., Arcoumanis, C., Gavaises, M., and Kampanis,N. Internal flow in Diesel injector nozzles: modelling and experiments In Proceedings of IMechE Seminar on Fuel Injection Systems, London, Paper S492/S2/99, 1999.

3. Payri, R., Salvador, F.J., Gimeno, J., and de la Morena, J. Macroscopic behavior of diesel sprays in the nearnozzle field. Presented at SAE World Congress, 2008-01-0929, 2008.

4. Oda, T., Goda, Y., Kanaike, S., Aoki, K., and Ohsawa, K. Experimental Study about internal Cavitating Flow and Primary Atomization of a Large-Scaled VCO Diesel Injector with Eccentric Needle 11th Triennial International Annual Conference on Liquid Atomization and Spray Systems, 132, 2009.

5. Chaves H., Knapp M., Kubitzek A., and Obermeier F., Experimental Study of Cavitation in the Nozzle Hole of Diesel Injectors Using Transparent Nozzles, SAE Paper No. 950290, 1995.

6. Chaves H., Kirmse C., and Obermeier F. The Influence of Nozzle Inlet Curvature on Unsteady Cavitation in Transparent Diesel Injection Nozzles, 1st International Colloquium on Microhydrodynamics, Paris, 2000.

7. Arcoumanis, C., Badami, M., Flora, H., and Gavaises M. Cavitation in Real-Size Multi-Hole Diesel Injector Nozzles. SAE Paper 2000-01-1249, 2000.

8. Soteriou, C., Andrews R.J., and Smith M., Direct Injection Diesel Sprays and the Effect of Cavitation and Hydraulic Flip on Atomization. SAE Paper 950080, 1995.

9. Walther J., Schaller J.K., Wirth R., Tropea C. Investigation of internal flow in transparent diesel injection nozzles using fluorescent particle image velocimetry (FPIV), Proc. ICLASS 2000, 2000.

10. Sou, A., Tomiyama, A., Hosokawa, S., Nigorikawa, S., and Maeda, T. Cavitation in a Two-Dimensional Nozzle and Liquid Jet Atomization, JSME International Journal Series B, 2006. 
11. Ganippa L.C., Bark G., Andersson S., Chomiak J., Comparison of cavitation phenomena in transparent scale-up single-hole diesel nozzles, Proc. CAV2001 A9.005, 2001.

12. Wollenhaupt M. et al., Springer Handbook of Lasers and Optics, edited by Träger F.,Springer, New York, 2007.

13. Malitson H., Refractive Index of Fused Silica, J. Opt. Soc. Am. 55, 1205, 1965.

14. Ciddor, P.E., Refractive index of air: new equations for the visible and near infrared, Appl. Optics 35, 1566$1573,1996$.

15. Bass, M., DeCusatis, C., and Enoch J. Handbook of Optics, 3rd edition, Vol. 4. McGraw-Hill 2009.

16. Kuiper, et al., Patent Aplication Publication, Publication №2008/0204890 A1, United States, 2008.

17. Deanesly, R.M. and Carleton I.T., Physical Constants of the Normal Paraffin Hydrocarbons. The Journal of Physical Chemistry, ACS Publications, 1941.

18. Ghosh, G., Handbook of Thermo-Optic Coefficients of Optical Materials with Applications Academic, San Diego, Calif., 1997.

19. Bach, H. and Neuroth, N. The Properties of Optical Glass Springer, New York, 1995.

20. Forziati, A.F., J. Research Null. Bur. Standards, 44, 373, 1950.

21. Scienceworld.wolfram.com. http://scienceworld.wolfram.com/physics/HartmannDispersionFormula.html 